\title{
Analysis of longitudinal beam dynamics behavior and rf system operative limits at high-beam currents in storage rings
}

\author{
T. Mastorides, ${ }^{*}$ C. Rivetta,${ }^{\dagger}$ J. D. Fox, and D. Van Winkle \\ Stanford Linear Accelerator Center, Stanford, California 94309, USA \\ D. Teytelman
}

Dimtel Inc., San Jose, California 95124, USA

(Received 29 December 2007; published 17 June 2008)

\begin{abstract}
A dynamics simulation model is used to estimate limits of performance of the positron-electron project (PEP-II). The simulation captures the dynamics and technical limitations of the low level radio frequency (LLRF) system, the high-power rf components, and the low-order mode coupled-bunch longitudinal beam dynamics. Simulation results showing the effect of nonlinearities on the LLRF loops, and studies of the effectiveness of technical component upgrades are reported, as well as a comparison of these results with PEP-II measurements. These studies have led to the estimation of limits and determining factors in the maximum stored current that the low energy ring/high energy ring (LER/HER) can achieve, based on system stability for different rf station configurations and upgrades. In particular, the feasibility of the PEP-II plans to achieve the final goal in luminosity, which required an increase of the beam currents to $4 \mathrm{~A}$ for LER and 2.2 A for HER, is studied. These currents are challenging in part because they would push the longitudinal low-order beam mode stability to the limit, and the klystron forward power past a level of satisfactory margin. An acceptable margin is defined in this paper, which in turn determines the corresponding klystron forward power limitation.
\end{abstract}

DOI: 10.1103/PhysRevSTAB.11.062802

PACS numbers: 29.20.-c, 29.27.Bd

\section{INTRODUCTION}

This paper follows the work previously presented by the authors in $[1,2]$. The first paper highlights the formalism and validation of the simulation tool, and presents preliminary growth rate estimates for the low energy ring (LER) for anticipated higher currents. Initial estimates for $\mathrm{rf}$ power and beam dynamics limits for both PEP-II rings have been addressed in [2].

This paper expands the analysis of rf power and beam dynamics limits and includes more conclusive validation data at higher currents. In particular, it focuses on the impact of increasing beam currents on the longitudinal stability boundaries imposed by the beam-cavity interaction. We study the implemented baseline low level radio frequency (LLRF) system as well as possible improvement via several technical component upgrades. These studies have provided important insight on LLRF configurations and operation point trade-offs for the PEP-II operations beginning December $2007 .^{1}$

This paper is organized as follows: Section II summarizes the PEP-II rf systems, the LLRF implementation, and the system features which implement the impedance con-

\footnotetext{
*themis@slac.stanford.edu

†rivetta@slac.stanford.edu

${ }^{1}$ Historically, a PEP-II run corresponds to roughly a year of operation. Run 5 covers operations from May 2005 to October 2005, run 5b from November 2005 to August 2006, run 6 from January 2007 to September 2007, and run 7 from December 2007 to April 2008.
}

trol feedback around the accelerating cavities. Sections III and IV present the limiting currents and LLRF configuration issues for the high energy ring (HER) and LER, respectively. Section V outlines the LLRF upgrades recently installed in PEP-II and presents their effect on the machine performance. Section VI analyzes the choice of the limiting conditions and their resulting quantitative limits used in this paper. Finally, Sec. VII summarizes our results and the conclusions for run 7 based on these studies.

\section{PEP-II RINGS-LONGITUDINAL SYSTEMS}

The PEP-II facility consists of two independent storage rings. The high energy ring ( $9-\mathrm{GeV}$ electron beam) and the low energy ring (3.1-GeV positron beam) each have harmonic number 3492 and operate at the rf frequency of $476 \mathrm{MHz}$. Continuous injection is achieved using collision energy electrons and positrons from the SLAC Linac and damping ring complex. The HER contains $11 \mathrm{rf}$ stations, while the LER is composed by 4 rf stations. Each PEP-II station has a 1.2 MW klystron. The klystrons are built by SLAC, Phillips and Marconi, and each design has different characteristics and performance.

Some klystrons power 2 normal-conducting rf cavities whereas others power 4 normal-conducting $\mathrm{rf}$ cavities. The rf cavities have high-order mode dampers and an $R / Q$ ratio of 116. The LLRF systems include direct and comb loop feedback paths to reduce impedances seen by the beam. A block diagram of one of the rf stations is shown in Fig. 1. A description of the feedback loops and their purpose, as well 


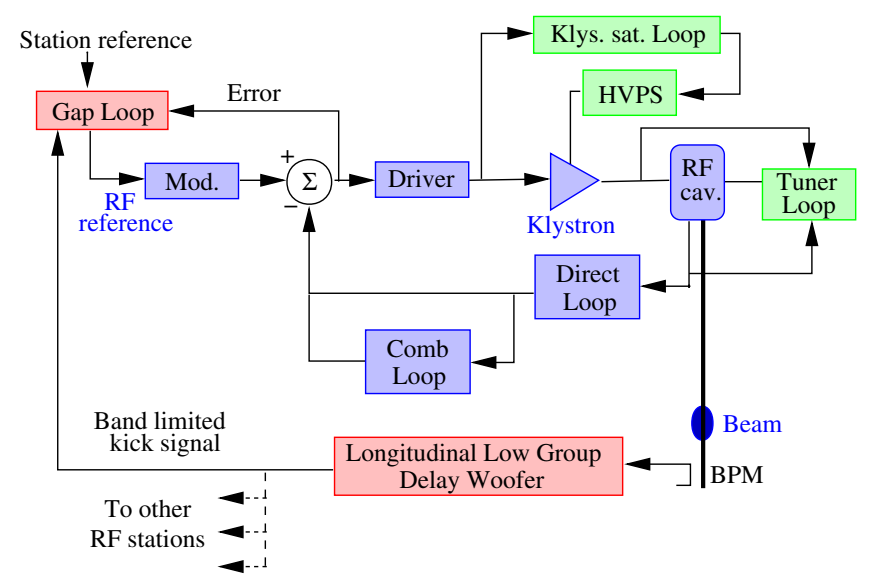

FIG. 1. (Color) The rf station block diagram.

as of the numerous low bandwidth regulating loops, can be found in [3].

In multibunch rings, the time (or phase) deviations of the bunch centroids, with respect to each bunches' equilibrium synchronous phase, can be transformed into a frequencydomain modal description. Typically we project this motion into the modal basis of a perfectly uniform even fill (the even-fill eigenmode basis) [4]. For the PEP-II case (with an rf frequency of $476 \mathrm{MHz}$, a revolution frequency of $136 \mathrm{kHz}$, and a harmonic number of 3492 buckets), a bytwo filling pattern yields 1746 filled bunches. The phase oscillations of these bunches can be decomposed into 1746 even-fill modes. Mode $n$ corresponds to a phase difference between adjacent bunches of $2 \pi(n / 1746)$, where $n$ ranges from 0 to 1745 [5]. Therefore, modes repeat with a period of 1746 , so that modes 873 to 1745 are equivalent to modes -873 to -1 . Thus, all the modes can be represented with the range from -873 to 872 . This modal representation has been used in this paper.

Coupled-bunch interactions between the beam and the longitudinal impedance of the rings are controlled via two strategies. The LLRF systems use direct and comb loop feedback [3] to reduce the effective impedance of the cavity fundamental. A band limited low group delay woofer (LGDW) system addresses the beam motion from in-cavity low-order modes via a signal from a beam pickup and control paths through the rf stations [6-9]. There is one LGDW channel for each PEP-II ring. A wideband bunch by bunch channel addresses all modes via a digital control filter and broadband longitudinal kickers, and is needed to control instabilities from the higher-order mode impedance [8].

The maximum achievable currents for the rings are limited by several mechanisms. Thermal effects from rf power, beam induced heating, and synchrotron radiation are limits addressed in the original design of the rf cavities, rf power elements, etc. Improvements of these systems are costly and difficult to implement, so upgrades to these systems are not considered in this study. Since commis- sioning, the number of rf cavities has increased from 16 to 28 in HER and from 4 to 8 in LER to provide accelerating voltages of 16-18.5 MV and 4.05-5.4 MV, respectively. Similarly, vacuum chambers and bellows have evolved during the operation of the facility since 1998 to anticipate a maximum beam current of $2.2 \mathrm{~A}$ in HER and $4 \mathrm{~A}$ in LER [10].

Increased stored currents affect the stability of the beam and the system's robustness to perturbations. In this paper, we analyze beam current limits induced by the low-order mode beam dynamics driven by the cavity fundamental mode. The dynamic interaction between the fundamental mode of the rf cavities and the beam becomes more unstable at increased currents, reaching the limit in the maximum damping the LGDW can provide. To reduce this interaction and minimize the growth rates of the fast beam mode dynamics, feedback loops are applied in the rf stations to reduce the effective impedance of the cavity as seen by the beam. The achievable minimum cavity impedance is limited by the klystron characteristics and rf station stability. Furthermore, the robustness to perturbations is related to the dynamic range of the klystron (critical operation point). Therefore, the stability and robustness of the system are directly impacted by the klystron transfer characteristics as will be further analyzed in Sec. VI.

\section{A. Klystron power requirements -impact of klystron characteristics}

The rf systems must satisfy several concurrent requirements. They must provide a specific accelerating voltage, deliver the necessary rf power to compensate synchrotron radiation and high-order mode losses, regulate the rf fields by filtering out perturbations and drifts, and minimize the cavity fundamental impedance. All of these requirements

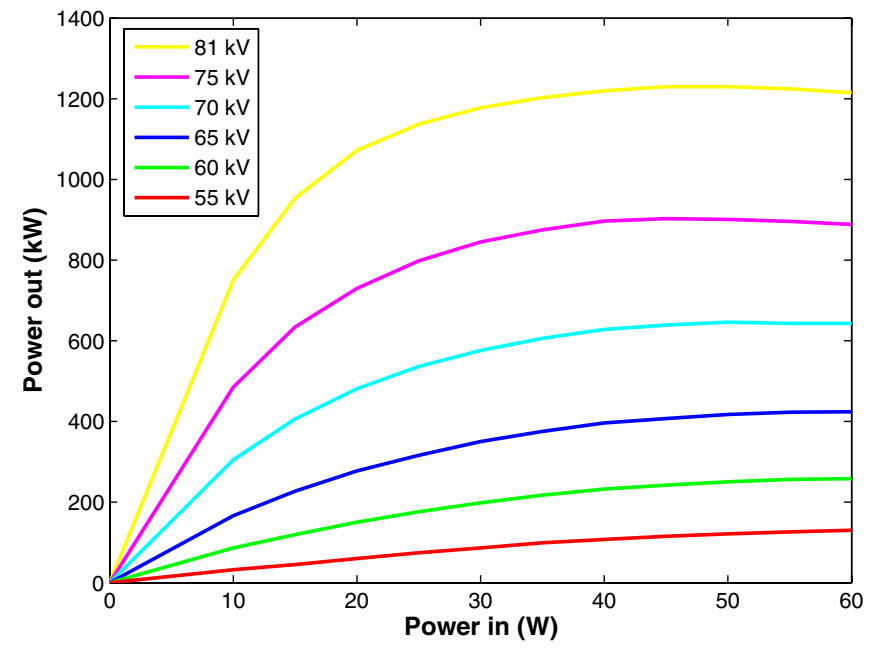

FIG. 2. (Color) Power curves for SLAC \#5 klystron. For a desired output power, the operation point is defined by the high voltage power supply level and the input power. 
are directly impacted by the base klystron power transfer characteristics as depicted in Fig. 2 through the power curves of a 1.2 MW SLAC klystron as used in the HER. It is important to notice from these curves that a specific output power requirement can be met over a range of high voltage power supply (HVPS) voltages and input rf powers. The choice though of the specific klystron operation point is very critical.

In operation the klystron average power limit must be set lower than the maximum specified power to accommodate high voltage power supply ripple, line perturbations which modulate the HVPS voltage, and klystron saturation effects that limit the klystron gain (as further analyzed in Sec. VI). The collective margin amounts to a $15 \%-20 \%$ reduction of the maximum available klystron forward power. These margins set the practical maximum steady state power limits to $\approx 1030 \mathrm{~kW}$ for SLAC klystrons and $\approx 930 \mathrm{~kW}$ for Phillips/Marconi klystrons.

In the HER, station 4-6 is implemented with a Phillips manufactured klystron and all the other stations are equipped with SLAC klystrons. In the LER, there are 2 SLAC, 1 Phillips and 1 Marconi, klystrons installed.

\section{B. Beam current limits due to low-mode instability growth rates}

At operating currents, the cavity-beam interaction is unstable due to longitudinal coupled instabilities in the absence of damping and rf station feedback loops. The strategy to achieve control of the cavity-driven modes uses the LLRF control loops to reduce the effective impedance seen by the beam (reducing the impedance driven growth rates). The reduced impedance may still excite unstable motion, so the LGDW woofer is then used to achieve final control. In the PEP-II implementation, it is not feasible to have acceptable control with only one of these control schemes due to the magnitude of the cavity impedance and the group delay limits on control channel gain.

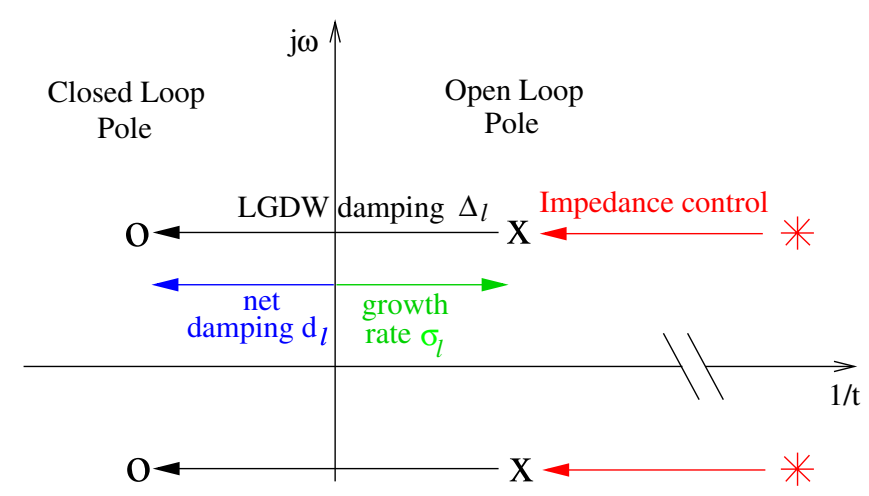

FIG. 3. (Color) Representation of the closed loop pole of the most unstable beam mode $(O)$, the open loop pole $(x)$ and the open loop pole in the absence of impedance control feedback (red *).
In Fig. 3, we qualitatively show this strategy via the eigenvalues of the most unstable low-order beam mode for the above condition (red *). It can be seen that the impedance control feedback loops in the rf station move these unstable eigenvalues close to the imaginary axis (black $\mathrm{x}$ ). For high-beam currents these dominant modes are still unstable. Application of the LGDW damping feedback channel stabilizes these modes (black $\bigcirc$ ).

In the simulation, we can estimate the magnitude of the eigenvalues of the most unstable low-order beam modes. The stability margins are then determined by comparing the simulated growth rates (real part of the eigenvalues) with the estimated maximum feedback induced damping rate $\Delta_{l}$ based on the LGDW channel configuration. Our stability margin criterion requires that the free growth rate $\sigma_{l}$ of the most unstable mode $l$ be lesser in magnitude than the net damping rate $d_{l}$ with feedback on $\left(\left|d_{l}\right| \geqq \sigma_{l}\right)$, as shown in Fig. 3. The margin is necessary to allow for fluctuations of the growth rates or reduction of the damping rates due to drifts of parameters. Since the net damping rate is the sum of the growth rate and the feedback induced damping,

$$
d_{l}=\sigma_{l}+\Delta_{l},
$$

the criterion is equivalent to the growth rate being smaller in magnitude than half the maximum available LGDW damping.

$$
\begin{aligned}
\left|d_{l}\right|>\sigma_{l} & \Rightarrow-d_{l}>\sigma_{l} \Rightarrow-\sigma_{l}-\Delta_{l}>\sigma_{l} \\
& \Rightarrow \sigma_{l}+\frac{1}{2} \Delta_{l}<0 .
\end{aligned}
$$

The maximum available LGDW damping rate for the HER is around $-3 \mathrm{~ms}^{-1}$ for the present configuration [7]. Therefore, for sufficient practical margins, the maximum operational growth rate is set to $1.5 \mathrm{~ms}^{-1}$. The maximum LGDW damping for the LER is $-6 \mathrm{~ms}^{-1}$ so that the maximum operational growth rate is $3 \mathrm{~ms}^{-1}$. Data for the LER damping rates has been presented in [1].

\section{HER LIMITATIONS}

\section{A. Beam current limits due to klystron power}

A dominant limitation for an increase of the HER beam current is the available forward klystron power. The HER contains $3 \mathrm{rf}$ stations with 4 cavities each and 8 rf stations with 2 cavities each for a total of 28 cavities. A careful balance of power between the 2 and 4 cavity stations is necessary to maximize the beam current. The power distribution depends on the cavity voltages and loading angles. In our analysis, we assume that the cavities in all the 2 cavity stations operate at the same voltage $V_{2}$ whereas the cavities in all the 4 cavity stations operate at the voltage $V_{4}$. This uniformity is necessary to avoid arcing limits.

From operational experience we also set $V_{2}=1.1 V_{4}$. It is then possible to determine the loading angle that maximizes the beam current for each gap voltage (the sum of 


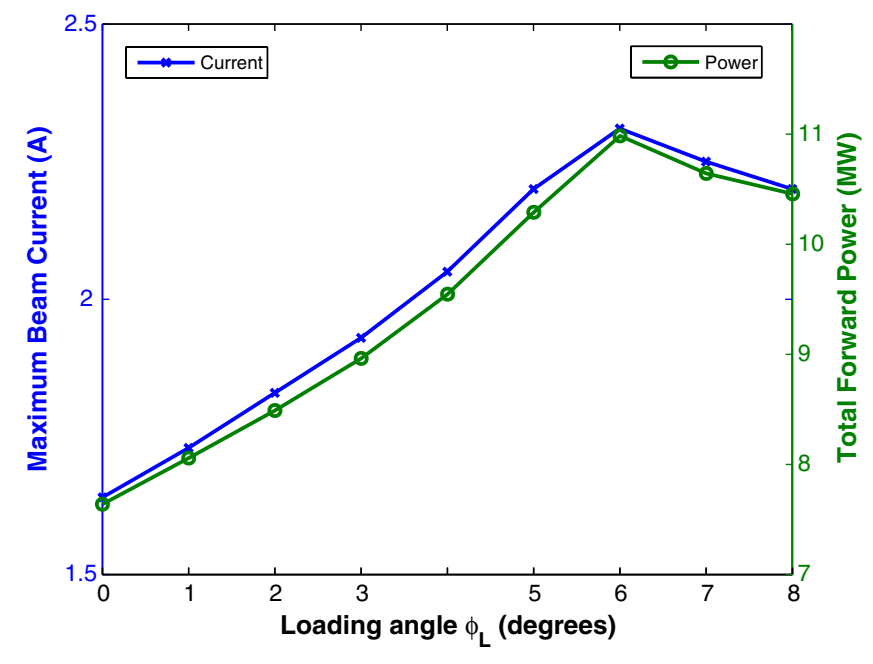

FIG. 4. (Color) HER maximum achievable beam current and total forward power as a function of the loading angle $\phi_{L}$.

the cavity voltages over all stations), based on the forward klystron power limit of $1030 \mathrm{~kW}$ per station and the reflected klystron power limit of $100 \mathrm{~kW}$ per cavity. The loading angle $\phi_{L}$ is defined as the total angle between the 2 cavity station voltage $V_{2}$ and the 4 cavity station voltage $V_{4}$. Essentially, the forward power of the 4 cavity stations is kept at the maximum and the loading angle is increased until the 2 cavity station forward or reflected power reaches its respective limit.

Figure 4 shows this optimization process for a gap voltage of $18.5 \mathrm{MV}$. As expected, the maximum beam current is achieved when the total forward power is at a maximum of $10.984 \mathrm{MW}$, near its limit of $11.33 \mathrm{MW}$. The limit of $11.33 \mathrm{MW}$ is achieved when all 11 stations operate at their maximum value of $1030 \mathrm{~kW}$. The maximum point defines the transition from the 4 cavity station limit to the 2 cavity station limit.

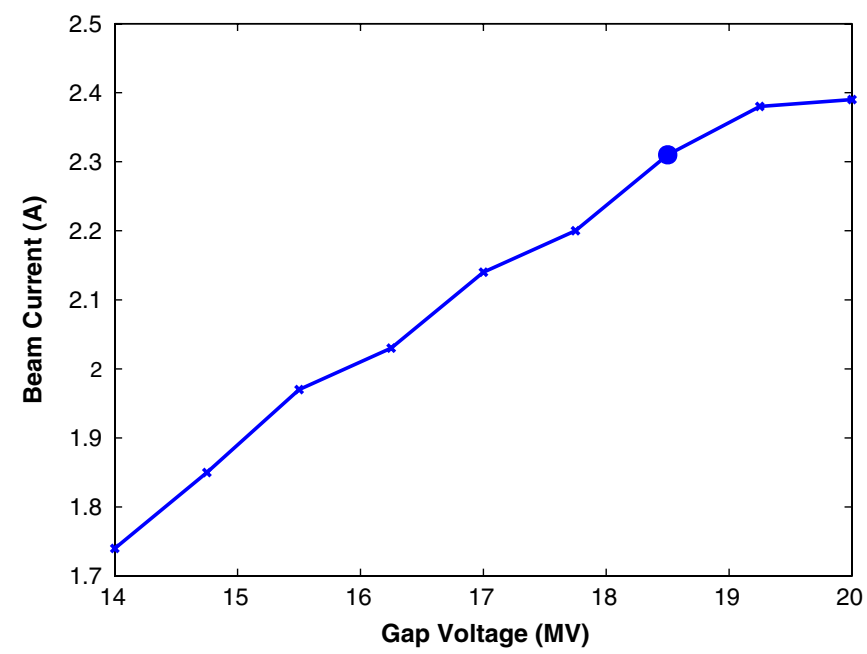

FIG. 5. (Color) HER maximum achievable beam current versus gap voltage. The $18.5 \mathrm{MV}$ operation point is marked with a circle.

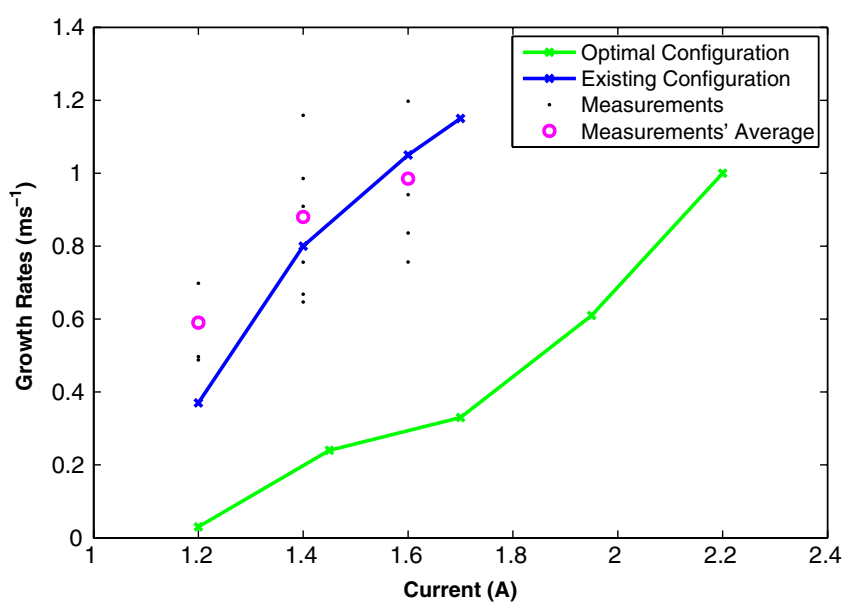

FIG. 6. (Color) HER growth rates of the most unstable low-order beam mode (mode -3 ).

By repeating the same analysis for various gap voltages, the maximum achievable current is plotted versus the gap voltage in Fig. 5. As expected, the maximum achievable current increases with gap voltage up to about $2.4 \mathrm{~A}$. At gap voltages above $18.5 \mathrm{MV}$ though, all 11 stations have to operate very close to their maximum forward power, leading to unsustainable operation. At the gap voltage of 18.5 MV - the highest considered for run 7-the maximum beam current is about 2.3 A with $1010 \mathrm{~kW}$ klystron forward power from each of the 4 cavity stations and $985 \mathrm{~kW}$ from each of the 2 cavity stations. These limits assume that all klystrons contribute equally to the beam power. If a station must run at reduced power (due to collector cooling limits, increased HVPS ripple margin, etc.) the achievable HER current must be consequently reduced.

\section{B. Beam current limits due to growth rates}

Figure 6 shows the growth rates for a gap voltage of $16 \mathrm{MV}$ (blue), as we presented them in [2]. These growth rates are estimated from the simulation time-domain data of beam motion, via transformation to the modal domain and exponential fitting to the modal amplitudes versus time. These are compared and show good agreement with the measured growth rates for the same configuration in the physical machine (magenta). It is also possible to observe the variance of the measured growth rates which necessitate the margins defined above. ${ }^{2}$ In the same plot, simu-

\footnotetext{
${ }^{2}$ A large component of the measurement scatter reflects actual variations of the effectiveness of the impedance control techniques over time. At any given operating point, the system is continuously perturbed and the instantaneous effective impedance fluctuates due to klystron power supply ripple, line transients, beam signals, etc., all contributing to an operating point modulation. As the growth measurements are unsynchronized to these perturbations, each measurement is a snapshot of some particular operating point and resulting effective impedance.
} 
lated data describing the performance of the HER operating at 18.5 MV is presented (green). This is the highest gap voltage that was considered for run 7 and shows a significant reduction of the growth rates. The 18.5 MV configuration also includes the improved klystron driver amplifiers which allow us to set the rf station feedback loop close to the optimal setting. The installation of klystron driver amplifiers with a more linear response is one of the technical upgrades implemented during run 6 , as will be shown in Sec. V.

From this curve, it is determined that the HER low-order mode growth rates do not exceed our estimated limit of $1.5 \mathrm{~ms}^{-1}$ due to LGDW damping, even for the highest planned current of 2.2 A with the gap voltage at $18.5 \mathrm{MV}$. As a result, the klystron forward power limit defines the maximum operational HER current to $2.3 \mathrm{~A}$ for $18.5 \mathrm{MV}$. With the run 6 gap voltage of $16 \mathrm{MV}$ both the klystron power and the growth rate limit are crossed at about $2.1 \mathrm{~A}$.

\section{LER LIMITATIONS}

\section{A. Beam current limits due to klystron power}

Figure 7 shows the required klystron forward power per station versus current for three different gap voltages in the LER. ${ }^{3}$ With the existing LER power configuration, the operational total forward power as defined in Sec. II is $2 \times$ $930 \mathrm{~kW}+2 \times 1030 \mathrm{~kW}=3920 \mathrm{~kW}$ or equivalently, the average forward power per station is $980 \mathrm{~kW}$. In Fig. 7, this limit is labeled "Run 6 Limit," while for comparison, the line "SLAC Limit" defines the maximum power per station if all the rf stations are equipped with SLAC klystrons. The limit is crossed at $3600 \mathrm{~mA}$ with $4.05 \mathrm{MV}$ gap voltage, at $3750 \mathrm{~mA}$ with $4.5 \mathrm{MV}$, and at $3950 \mathrm{~mA}$ with $5 \mathrm{MV}^{4}{ }^{4}$

During run 6 the gap voltage was set at $4.05 \mathrm{MV}$, whereas for run 7 the highest considered gap voltage was $5 \mathrm{MV}$, assuming no vacuum chamber heating problems [10]. If all klystrons in the LER were to be SLAC klystrons, the limits from power considerations become 3750 and $3900 \mathrm{~mA}$, respectively, for 4 and $4.5 \mathrm{MV}$. A gap voltage of at least $5 \mathrm{MV}$ would be necessary to reach $4 \mathrm{~A}$ in the LER.

In Fig. 7 power measurements from the machine during run 6 [11] are also plotted to check the accuracy of the estimation. The theoretical estimates and the measurements have the same general form. The small difference between them could be attributed to possible calibration issues during the experiments or differences between de-

\footnotetext{
${ }^{3}$ We have presented much of the data in Figs. 7, 8, 9, and 12 in [2], but have included it in this paper with a more complete description of the LER limitations.

${ }^{4}$ The necessary klystron forward power for a given current decreases with increased gap voltage due to cavity overcoupling. PEP-II cavities were designed for $750 \mathrm{kV}$ nominal voltage and are operating at $500-625 \mathrm{kV}$.
}

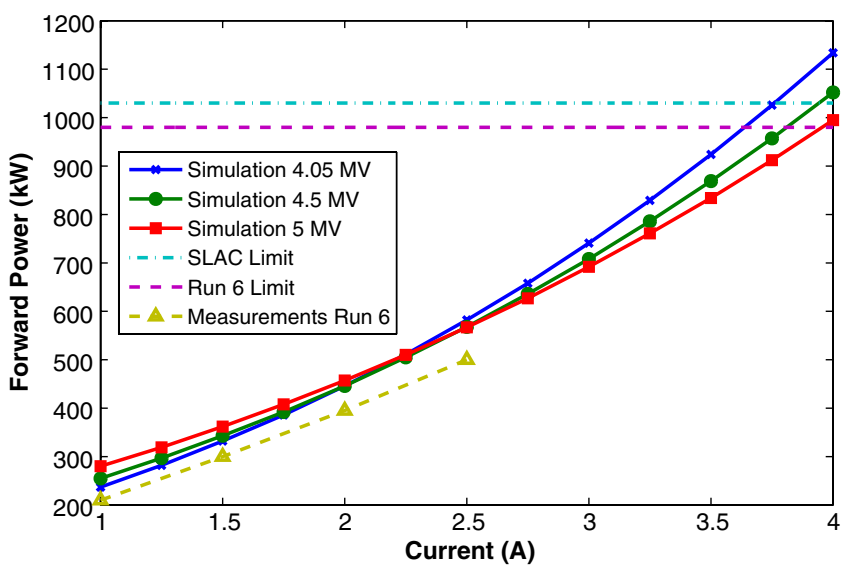

FIG. 7. (Color) LER klystron forward power versus current.

sign report values used in the simulation [12] and the actual machine parameters.

To further examine the feasibility of the 4 A LER operation and to provide insight on the gap voltage dependence and margin, the forward power is plotted versus the gap voltage in Fig. 8. From this plot, one can see that at least 4.05 MV are needed for 3.6 A. With a gap voltage of $5 \mathrm{MV}$, operation will be marginal and unreliable at $4 \mathrm{~A}$, unless four SLAC klystrons are used in the LER (which were not considered for run 7). A gap voltage of at least 5.5 MV is required for sufficient margins at $4 \mathrm{~A}$. At the increased gap voltage of $5 \mathrm{MV}$ and higher, problems with vacuum chamber heating and high-order mode structural resonance issues related to the shorter bunch length may arise [10].

\section{B. Beam current limits due to growth rates}

The LER coupled-bunch instabilities due to the rf cavity fundamental impedance are a significant limitation to the maximum attainable current. Therefore, possible upgrade scenarios were studied to determine what improvements would be necessary to reach the planned 4 A beam current

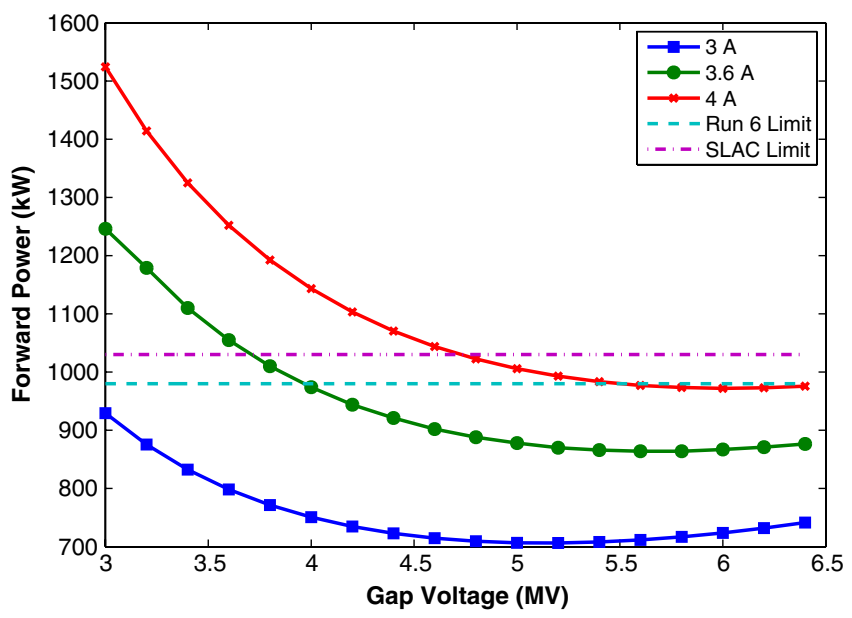

FIG. 8. (Color) LER klystron forward power versus gap voltage. 


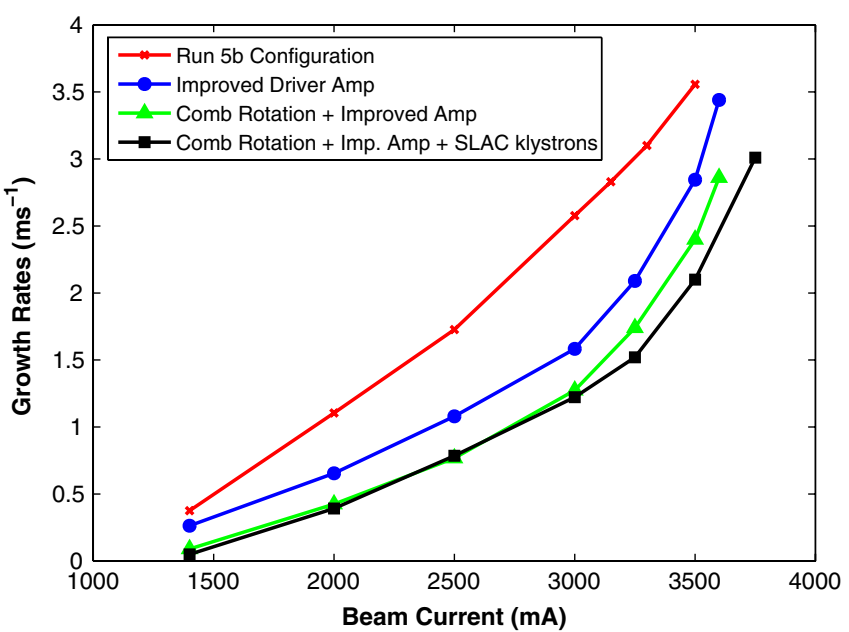

FIG. 9. (Color) Estimated growth rates at $4.05 \mathrm{MV}$.

for run 7. The upgrades that can significantly improve the limit include operation with four SLAC klystrons, the use of more linear LLRF drive amplifiers [13], and the full implementation of the comb phase rotation (a partially implemented trade-off of $\mathrm{rf}$ station stability to growth rate improvement further discussed in Sec. V) [1].

Figure 9 presents the simulated growth rates for the dominant unstable beam mode in the LER (mode -3 ). It depicts various LLRF configurations with LER operating at 4.05 MV gap voltage. The red curve presents the LLRF configuration used during run $5 \mathrm{~b}$, before any of the technical upgrades were implemented. The blue curve shows the reduction in the growth rates using the more linear driver amplifiers (improved driver amplifier). The green curve is a configuration with the improved amplifiers and comb rotation implemented. Finally, the black curve additionally assumes four SLAC klystrons in the LER.

As the current goes up, the klystron gain is reduced, especially when the HVPS has reached its maximum (highest curve in Fig. 2). To sustain the same impedance control performance, the total loop gain is kept constant by increasing the feedback gain up to its maximum value set by the stability of the loop. Past this point, the effectiveness of the impedance control loop is reduced, leading to an increase in the growth rate slope, as seen after about $3000 \mathrm{~mA}$.

In Sec. II and in [1], the stability criterion was defined to set the absolute value of the net damping rate higher than the growth rate so that the system is not only stable, but also immune to perturbations and drifts of parameters. Defining $m_{l}$ as the damping margin,

$$
\left|d_{l}\right|>\sigma_{l} \Rightarrow-d_{l}>\sigma_{l} \Rightarrow m_{l}=\sigma_{l}+d_{l}<0,
$$

to check for the stability margin according to our criterion, we can plot the damping margin and look for the zero crossing. The damping margin is shown in Fig. 10.

Figure 11 shows the damping margin for a gap voltage of 4.05 MV. The inflection point at $3000 \mathrm{~mA}$ in Fig. 11 is

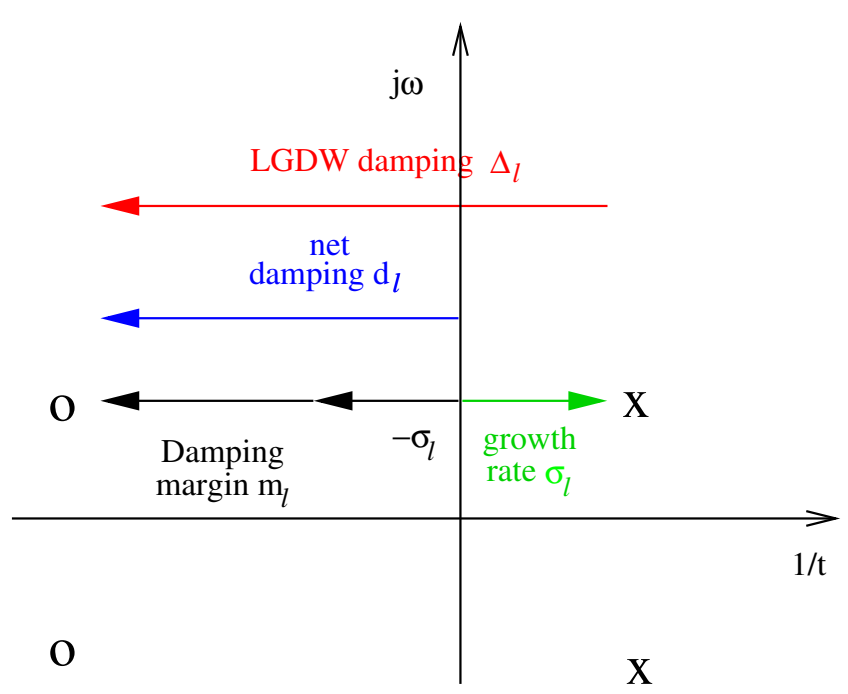

FIG. 10. (Color) Damping margin definition.

partly due to the small increase in the slope of the growth rates, but mostly to a limitation of the LGDW channel's damping due to the group delay limit of its gain.

From Figs. 9 and 11, we can predict that the limit due to growth rates for the LER operating at $4.05 \mathrm{MV}$ is at about $3150 \mathrm{~mA}$ with the run $5 \mathrm{~b}$ rf station implementation, and at $3525 \mathrm{~mA}$ with the improved amplifiers. The current limit of $3600 \mathrm{~mA}$, set by the klystron forward power, can be reached with the combination of the improved amplifiers and comb rotation, since the growth rates still have sufficient margin at this point. The maximum achievable current with $4.05 \mathrm{MV}$ is $3750 \mathrm{~mA}$ with all four SLAC klystrons and both upgrades implemented.

Figures 12 and 13 present the same configurations as Figs. 9 and 11, but for the higher gap voltage of $4.5 \mathrm{MV}$. The increase of gap voltage to $4.5 \mathrm{MV}$ raises the limit of the run $5 \mathrm{~b}$ configuration to $3350 \mathrm{~mA}$ and with the improved amplifiers to $3725 \mathrm{~mA}$. A combination of the improve-

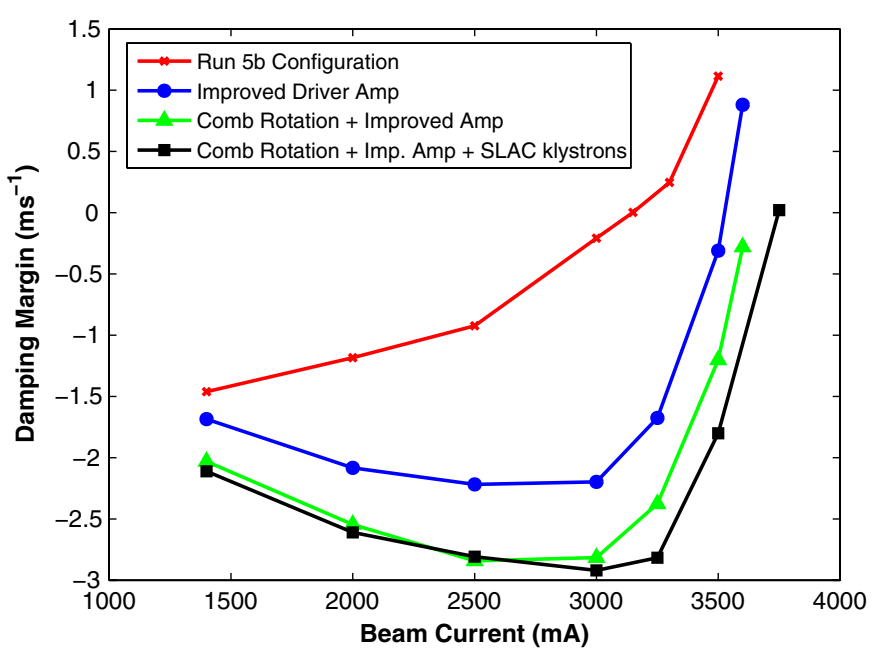

FIG. 11. (Color) Estimated damping margins at 4.05 MV. 


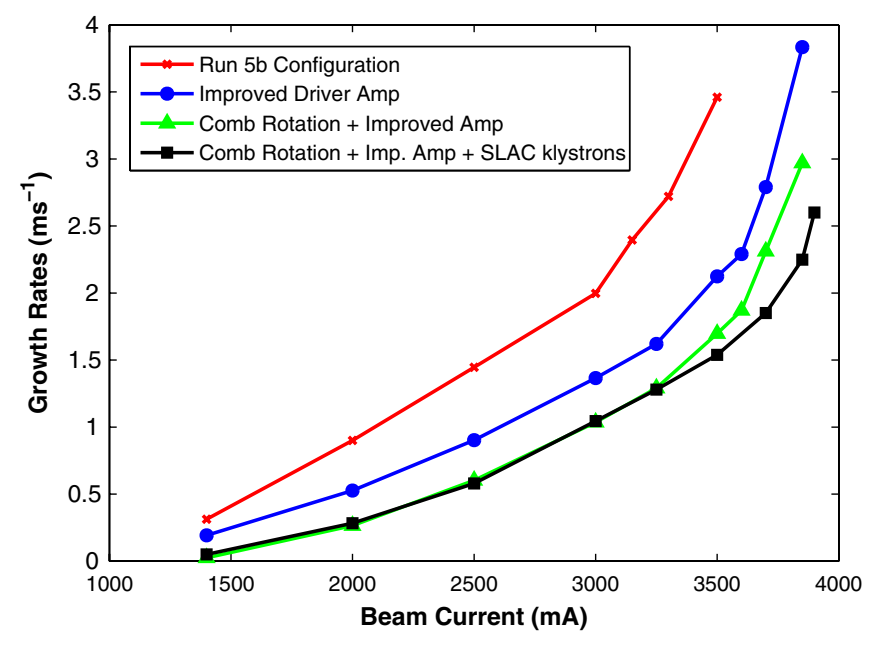

FIG. 12. (Color) Estimated growth rates at $4.5 \mathrm{MV}$.

ments achieves the power limit of $3750 \mathrm{~mA}$ with two SLAC klystrons or $3900 \mathrm{~mA}$ with four SLAC klystrons.

The studies above have shown that a gap voltage of at least $5 \mathrm{MV}$ would be necessary to reach $4 \mathrm{~A}$ in the LER according to the klystron power criterion. Figures 14 and 15 show the improvement of growth rates with increasing gap voltage but also the further reduction of growth rates with the full implementation of the comb rotation. The gap voltage is set to $4.05,4.5$, and $5 \mathrm{MV}$ for the red, blue, and green curves, respectively. The simulated configuration for these curves is that of the end of run 6 with the improved amplifiers and a partial comb rotation of $10^{\circ}$. The magenta curve is also at $5 \mathrm{MV}$, but with the optimal comb rotation of $20^{\circ}$ implemented, as we had planned for run 7 . The early termination of run 7 did not allow us to implement the optimal comb rotation during normal operations, but only during testing. The reduction of the growth rate with gap voltage is obvious. One can also see that the growth rates for the $5 \mathrm{MV}$ case at $4 \mathrm{~A}$ are significantly lower than the

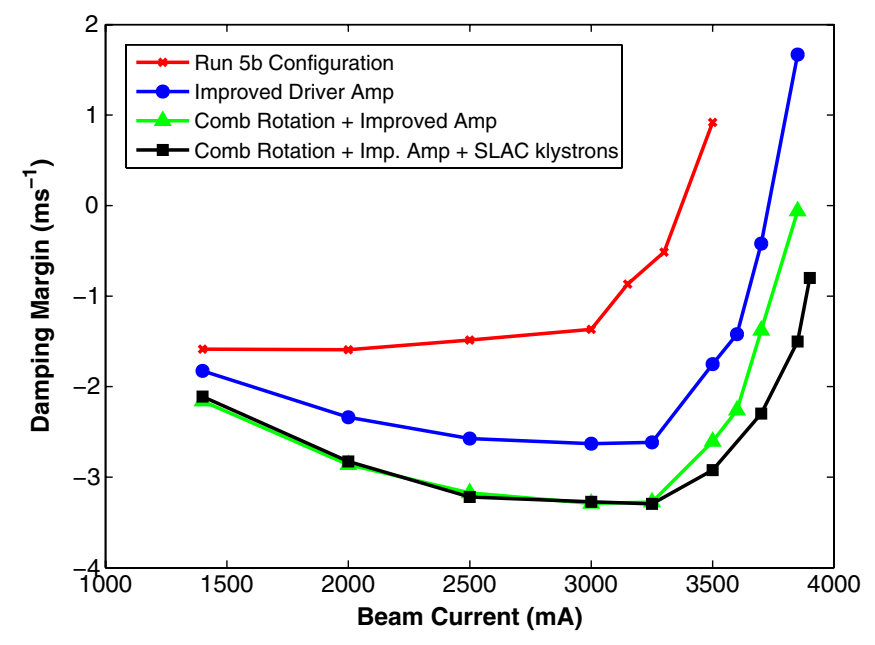

FIG. 13. (Color) Estimated damping margins at 4.5 MV.

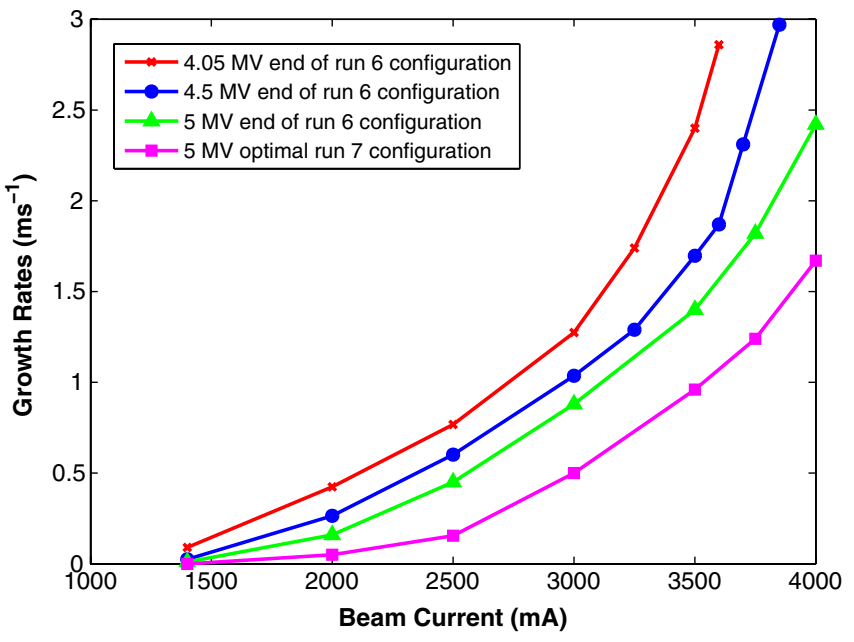

FIG. 14. (Color) Comparison of simulation growth rates for different gap voltage settings.

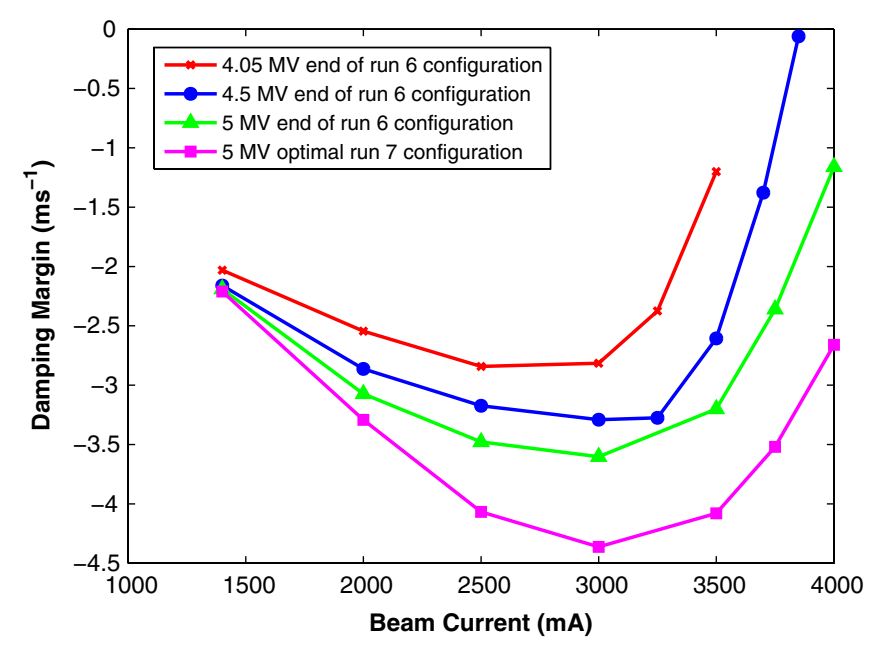

FIG. 15. (Color) Comparison of estimated damping margins for different gap voltage settings.

$3 \mathrm{~ms}^{-1}$ limit, so this current is not limited by beam stability if there is sufficient klystron power.

These results are summarized in Table I, where the limiting factor (beam stability through growth rates or available klystron power) and the corresponding current is presented for each configuration. From this summary, one can see that the LER situation is more difficult because both limitations are reached. Higher gap voltages will be necessary, which might cause vacuum chamber heating and high-order mode structural resonance problems [10]. Trade-offs between the limitations will be necessary at high currents.

\section{TECHNICAL UPGRADES' EFFECT ON REAL MACHINE PERFORMANCE}

As discussed in [1], during the development of the simulation, imperfections of the technical components of 
TABLE I. LER limiting factors and maximum current for each configuration. The $\sqrt{ }$ mark signifies the applied upgrades in each configuration whereas the SLAC klystrons column shows how many of the four LER stations have SLAC klystrons installed (2 SLAC klystrons at the end of PEP-II operations).

\begin{tabular}{|c|c|c|c|c|c|}
\hline Gap voltage & SLAC klystrons & Improved amps & Comb rotation & Limitation & Limit (mA) \\
\hline $4.05 \mathrm{MV}$ & 2 & $\cdots$ & $\cdots$ & Growth rate & 3150 \\
\hline $4.05 \mathrm{MV}$ & 2 & $\sqrt{ }$ & $\cdots$ & Growth rate & 3525 \\
\hline $4.05 \mathrm{MV}$ & 2 & $\sqrt{ }$ & $\sqrt{ }$ & Power & 3600 \\
\hline $4.05 \mathrm{MV}$ & 4 & J & $\sqrt{ }$ & Both & 3750 \\
\hline $4.5 \mathrm{MV}$ & 2 & $\cdots$ & $\cdots$ & Growth rate & 3350 \\
\hline $4.5 \mathrm{MV}$ & 2 & $\sqrt{ }$ & $\cdots$ & Growth rate & 3725 \\
\hline $4.5 \mathrm{MV}$ & 2 & $\sqrt{ }$ & $\sqrt{ }$ & Power & 3750 \\
\hline $4.5 \mathrm{MV}$ & 4 & $\sqrt{ }$ & $\sqrt{ }$ & Power & 3900 \\
\hline $5 \mathrm{MV}$ & 2 & $\sqrt{ }$ & $\sqrt{ }$ & Power & 3950 \\
\hline $5 \mathrm{MV}$ & 4 & $\sqrt{ }$ & $\sqrt{ }$ & $\cdots$ & $>4 \mathrm{~A}$ \\
\hline
\end{tabular}

the LLRF system were discovered and several technical upgrades to the system were evaluated. In Secs. III and IV, estimated growth rates from simulations were presented to evaluate the differences among the upgrade scenarios.

The replacement of the LLRF system $120 \mathrm{~W}$ solid-state driver amplifier is one of the upgrades. The nonlinear behavior of the old amplifiers introduced distortion in the small-signal transfer function [13]. This distortion did not allow the optimal setting of the parameters in the LLRF impedance control feedback loops, thus leading to an increase in growth rates. This behavior was predicted by the simulation before it was measured in the actual machine, highlighting the value of the simulation model in understanding the behavior of the physical system. Through the simulation it was shown that better amplifiers could not only reduce the growth rates but also improve the stability margins of the station. From these studies better linear driver amplifiers were identified, ordered, and installed. The testing methods and the characteristics of the selected amplifiers are described in [13].

Based on the analysis of the LLRF system parameter sensitivities as discussed in [1], we were able to determine that it is possible to achieve great improvement in the growth rates (exceeding 50\%) with a relatively small reduction in the LLRF loop stability margins - in particular the rotation of the comb loop phase by just $10^{\circ}$. Following these studies, a comb phase rotation has been applied since April 2006 in the LER rf system, allowing an increased beam stability margin.

After implementing these upgrades, experiments were conducted during runs 6 and 7 to prove their effectiveness and demonstrate the value of the simulation tool and its agreement with the physical system, up to the maximum current reached in the LER. Figure 16 compares the most unstable growth rates (mode -3 ) from simulated data (solid lines) and measurements from the machine (dashed lines) for the LER at 4.05 MV. From the simulated data, the red curve (circle markers) corresponds to the original system configuration used during run $5 \mathrm{~b}$ (as shown in Fig. 9), whereas the green curve (square markers) shows a configuration similar to the end of run 6 , with the improved amplifiers and the partial implementation of the comb rotation. The magenta curve (x markers) corresponds to a configuration with the improved amplifiers and the full comb rotation, that we had planned to use during run 7 . The first set of measurements in the red dashed line (circle markers) represents a configuration similar to the one used during run $5 \mathrm{~b}$. The blue (triangle markers) dashed curve corresponds to the run $5 \mathrm{~b}$ configuration after replacing the most distorted of the amplifiers (LER station 4-2). The black (diamond markers) dashed curve shows data taken at the end of run 6 , with the improved amplifiers and partial implementation of the comb rotation, but

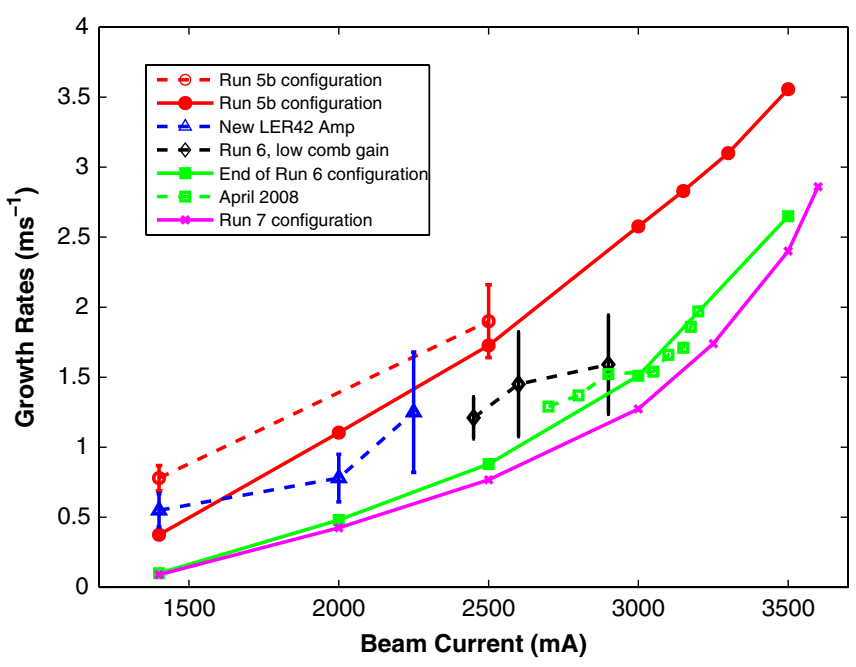

FIG. 16. (Color) Predicted (solid lines) and measured (dashed lines) growth rates. Improvements due to the upgrades are visible. Error bars are not included for the April 2008 data because they crowd the image. They are comparable in magnitude with the error bars from the run 6 data. 
slightly reduced comb loop gain. Finally, the green dashed data curve (square markers) corresponds to measurements from the last two days of PEP-II operations, when record LER currents were achieved, with the same configuration as in the end of run 6 (improved amplifiers, partial comb rotation). These measurements show impressive agreement with the predictions for this configuration.

Comparing the red and blue dashed curves, one can see the improvement by simply changing the most distorted of the amplifiers. The blue curve's growth rate measurement at $1400 \mathrm{~mA}$ is inaccurate mainly due to the short $3.5 \mathrm{~ms}$ measurement interval used, which does not allow for accurate fitting of a slowly growing exponential to the experimental data. The comparison of the green and red solid lines shows the substantial improvement expected from the implementation of the improved amplifiers and partial comb rotation by the end of run 6 . The similar comparison of the green and red dashed lines validates the simulation expectations, with the blue line providing the intermediate improvement with the replacement of the worst amplifier. The black and the green dashed line share the same configuration. Their difference is partially attributed to a slightly suboptimal setting of the comb loop gain-showing the system sensitivity to the operation point parameters-and might be also related to the problematic operation of an rf klystron power supply at the time. One should note the large error bars of the measured growth rates - a reality of the machine that further explains the more conservative definition of limits for our predictions.

It should be noted that at the end of run 6 , with the improved amplifiers installed and the comb rotation partially implemented $\left(10^{\circ}\right.$ rather than the optimal $\left.20^{\circ}\right)$, the peak LER current achieved in the actual machine was $3000 \mathrm{~mA}$ for a short period of time. Growth rates were not measured at this current, but only up to $2900 \mathrm{~mA}$. This limit was not imposed by the rf system, but it was related to aborts triggered by the detector radiation protection, and the restricted HER beam currents that reduced the beambeam stability effects.

Any disagreements of the estimated and measured curves can be partly attributed to the big variance of the measured data, but also to the fact that the simulation data are based on an ideal driver amplifier and do not present an exact representation of the system. The improved amplifiers greatly boost the system performance, but are still not ideal. Based on initial measurements, the amplifier nonlinearity, added to the distortion and nonuniformity of the radio frequency processor module in the LLRF control loop (RFP) [3], could possibly impact the effectiveness of the impedance control.

The magenta curve shows the maximum possible improvement with the upgrades as we were hoping to operate during run 7. Unfortunately, the early termination of PEP-II operations did not allow us to implement the full amount of comb rotation and measure this further improvement in the real system. The close agreement of simulated and measured data though, makes us confident that we would have achieved the additional improvement shown in Fig. 16.

The nonlinearity of the original amplifiers did not allow us to use the optimal setting of the impedance control feedback leading to a gain reduction of the feedback loop, as described earlier in this section. In runs 1 to 6 , the direct loop gain was set $3 \mathrm{~dB}$ lower than the optimal, due to this amplifier distortion. As the improved amplifiers were installed the direct loop gain was increased by $1.5 \mathrm{~dB}$ to test the performance of the system. An optimal setting of the gain via a further $1.5 \mathrm{~dB}$ gain increase planned for run 7 would have further reduced the growth rates as estimated by the magenta curve.

The amplifier distortion also prevented us from fully implementing the comb rotation to the estimated optimal $20^{\circ}$, especially at higher currents. The ability to now fully apply the comb rotation provides the means to verify the simulated prediction of growth rate reduction at higher currents (low current verification in [1]). Figure 17 shows the measured growth rate as a function of comb rotation for the LER at $2450 \mathrm{~mA}$ and compares it with the simulation. Both the measured and the simulated growth rate decrease with comb phase rotation, as predicted. The simulated and measured data have the same functional form and slope proving the value of the tool. The simulation is an idealized system and underestimates the growth rates due to residual imperfections of the LLRF from the driver amplifiers and the RFP module as described above.

Additional evidence of the distortion reduction with the improved amplifiers is shown in Fig. 18, where we compare the LER 4-5 closed loop transfer functions (measured as shown in $[1,14]$ ) for $0^{\circ}$ and $25^{\circ}$ comb rotation at $1200 \mathrm{~mA}$. Because of the significant distortion of the original amplifiers, the stability margin of the rf station feedback loops was greatly reduced. This effect can be seen by the peaks in the closed loop transfer function near

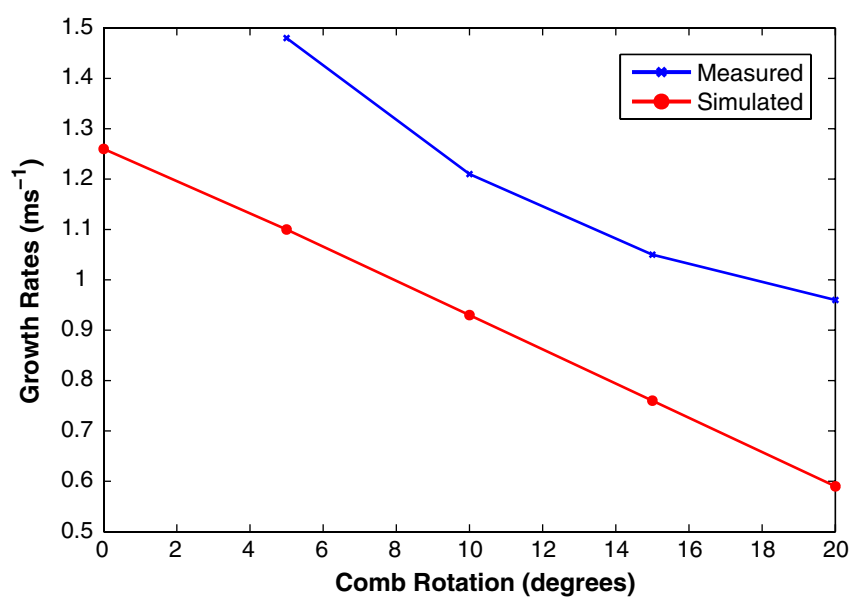

FIG. 17. (Color) Measured improvement in growth rates versus comb rotation for LER at $2450 \mathrm{~mA}$. 


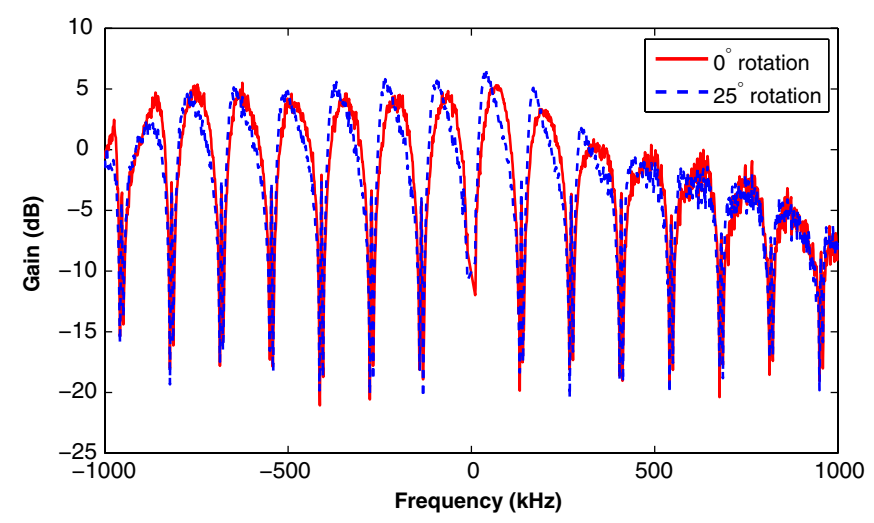

FIG. 18. (Color) Measured transfer function magnitude from LER 4-5 at $1200 \mathrm{~mA}$ showing minimal comb rotation induced distortion with the improved amplifiers.

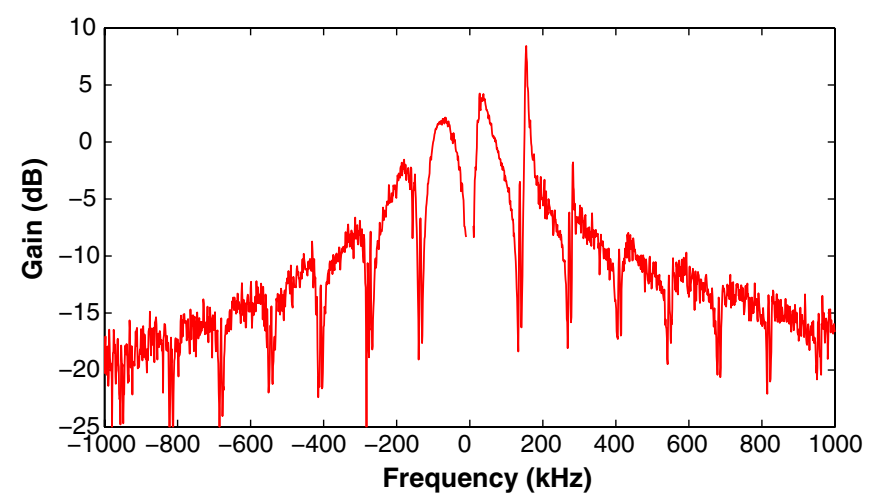

FIG. 19. (Color) Measured transfer function magnitude from LER 4-2 at $1500 \mathrm{~mA}$ during run $5 \mathrm{~b}$, showing comb rotation induced distortion with the original amplifiers.

the carrier frequency, as depicted in Fig. 19 for $20^{\circ} \mathrm{comb}$ rotation at $1500 \mathrm{~mA}$. The distortion resulted in reduced gain and phase margins and station instability. The improved amplifiers not only improve station stability but also allow us to implement the optimal value of the comb rotation for maximum growth rate reduction. It should be noted that, for the initial implementation at the beginning of run 6 , the comb rotation was limited to $10^{\circ}$ or even less for the PEP-II rf stations with the driver amplifiers presenting the highest distortion (for example LER station 4-2).

\section{ESTIMATION OF OPERATIONAL LIMITS}

The maximum current that the LER-HER can reach for various configurations has been estimated by analyzing the behavior and stability of the low-order beam dynamics. Criteria for a quantitative determination of the limiting conditions were briefly described in Sec. II. These criteria defined the growth rate and klystron power limits used in this paper. A more detailed discussion of these criteria follows.

In a dynamic system, as a particle accelerator, the operation point must be a stable equilibrium point. For a given stable operation point, there is a bounded space where the state variables of the system can be perturbed and a bounded system trajectory returns back to the original operation point. It is important to note that, even for a stable operation point, the system can lose local stability if a perturbation transiently moves the operation point away from this bounded space. Therefore, knowledge of the system parameters and nonlinearities that define stability and robustness to perturbations is necessary to determine the optimal operating point.

\section{A. Operation point stability}

The interaction between the beam dynamics and the cavity impedance makes the equilibrium point unstable in absence of feedback damping at the operating currents in PEP-II. The main source of instability is the rf cavity impedance, which destabilizes beam modes from -10 to +10 . To reduce instability, the station impedance is minimized for the low-order beam modes using impedance control feedback in a configuration combining direct and comb filter loops. The design goal is to reduce the interaction between the beam and the fundamental cavity impedance thus minimizing the growth rates of the fastest unstable modes (usually modes -3 or -4 depending on the operation point). The gain of the feedback loops though is limited by the klystron saturation characteristics.

The beam is stable only if the low-mode longitudinal LGDW channel can provide sufficient damping. The gain of the LGDW is limited by the group delay ${ }^{5}$ and the high frequency characteristics of the control filters. The maximum gain limit for the current configuration is reached at currents higher than $3 \mathrm{~A}$ in the LER.

From this description of the beam dynamics/cavity impedance interaction, it is important to notice that the loworder beam mode stability depends on the design of the LGDW and a careful design of the feedback systems constituting the impedance control of the rf station. For an optimal configuration of these systems, the beam stability is ultimately limited by the klystron characteristics.

\section{B. Operation point robustness to perturbations}

For the control of low-order mode beam motion, the robustness of the system to perturbations is associated with the maximum transient forward power that the klystron can apply to the cavities. As it was summarized in Sec. II, this maximum value depends not only on the maximum power that the klystron can dissipate but also on the saturation characteristics.

\footnotetext{
${ }^{5}$ The control filter in the LGDW is a programmable FIR filter with a $9.89 \mathrm{MHz}$ sampling rate (72 samples/turn) and up to 32 FIR coefficients per macrobunch. The control filters employed have an effective group delay of $66 \mu \mathrm{s}$ (HER) and $108 \mu \mathrm{s}$ (LER). Filters with narrower bandwidth (greater noise rejection) could have group delays up to $141 \mu \mathrm{s}[7,9]$.
} 
The different power out/in characteristics in Fig. 2 correspond to settings of the klystron HVPS. To run consistently in a relatively linear region, the input power is kept in the range between 15 and $20 \mathrm{~W}$ for all PEP-II klystrons, independent of the manufacturer. This is achieved by the klystron saturation loop of Fig. 1 which regulates the HVPS value based on the average value of the klystron input power. As greater power is demanded, the HVPS level is increased (increasing the large signal gain of the klystron), essentially moving the operation point vertically on the plot up to about $900 \mathrm{~kW}$ when the maximum HVPS voltage is reached. A further increase of output power above $900 \mathrm{~kW}$ requires increases in input power and leads into saturation, which effectively changes the amplitude and phase modulation gains differently.

Therefore, an increase in the input power for a constant forward power leads to a lower transient maximum power and margin. The maximum klystron forward power is operationally unsustainable since the small-signal gain goes to zero. The klystron saturation limits the klystron power margin and is significant in defining the robustness to perturbations around a given operation point.

The klystron saturation also reduces the effective gain of the system, leading to reduced impedance control through the feedback loops. To achieve a compromise between the saturation effects and the available power, the maximum operable klystron forward power is decreased by $15 \%-$ $20 \%$ from the maximum klystron power (as seen in the analysis in Sec. II). Effectively, this choice reduces the power efficiency in favor of improved impedance control and increased robustness to perturbations.

This trade-off has been studied using the simulation, but has also been demonstrated in the real machine. As an example, the peak current reached in the LER was approximately $3 \mathrm{~A}$ for runs $5 \mathrm{~b}, 6$, and 7 . For the first case, a small increase of the current to approach $3 \mathrm{~A}$ led to a considerable increase in the rate of aborts. On the other hand, at the end of run 6 and during run 7 the klystron operating point was set quite differently, so that technical issues (rather than rf margins) prevented a further increase of the current. This comparison shows how careful tuning of the rf stations and optimal choice of operating point can provide much higher margins, increase the robustness to perturbations, and considerably decrease the rate of aborts.

\section{CONCLUSIONS}

The simulation of the PEP-II rings described in this work is a close representation of the actual system. It has been used to study the existing system and planned upgrades. It has also been used to study and develop new operating configurations at multiples of the original design currents, as a means of better understanding operational strategies and ultimate limits of the systems. The insight gained from these studies has been very helpful in the effort to optimally use the resources and available hardware to achieve the highest possible currents.

Through this work, limiting factors and acceptable margins for high-beam current operations have been specified. The importance of the rf feedback loop configurations, the LGDW design, the klystron characteristics, and the dynamic stability margin has been better illustrated. In particular, it has been shown that the klystron saturation not only limits the klystron power margin, but also is essential in defining the robustness to perturbations around a given operation point.

These studies suggest that the HER could achieve 2.2 A with a gap voltage around $18.5 \mathrm{MV}$, but even slightly higher currents would be very difficult to reach due to klystron power limitations. For the LER with the LLRF station implementation from the beginning of run 6 and an increased gap voltage of $4.5 \mathrm{MV}$ the limit is at $3350 \mathrm{~mA}$. The improved amplifiers and the comb rotation as implemented by the end of run 6 raise this limit to $3750 \mathrm{~mA}$. Finally, SLAC klystrons for all LER stations and a gap voltage higher than $5 \mathrm{MV}$ would be necessary for $4 \mathrm{~A}$, with possible problems with vacuum chamber heating and issues related to higher order modes.

One of the most important features of the PEP-II timedomain model used in this work is the adaptability to simulate the interaction between the rf stations and the beam for other systems and accelerators. While motivated by PEP-II concerns, the simulation has been used to study Robinson instability [15] for SPEAR and will be adapted and enhanced for precommissioning studies of the Large Hadron Collider (LHC) at CERN.

\section{ACKNOWLEDGMENTS}

The authors would like to thank the SLAC klystron department for their help with klystron tests and the PEPII operations group for providing us with machine time for measurements. We would also like to thank Heinz Schwarz and Alexander Novokhatski for the helpful discussions of static power limits, as well as this paper's reviewers for their detailed and thorough comments that helped substantially improve this work. This work was supported by the U.S. Department of Energy under Contract No. DE-AC02$76 \mathrm{SF} 00515$.

[1] C. Rivetta et al., Phys. Rev. ST Accel. Beams 10, 022801 (2007).

[2] T. Mastorides et al., in Proceedings of the IEEE Particle Accelerator Conference, Albuquerque, NM, 2007; SLACPUB-12654.

[3] P. Corredoura, Report No. SLAC-PUB-8124, 1999.

[4] S. Prabhakar, Ph.D. thesis, Stanford University Applied Physics Department, 2001; SLAC-Report-554, 2001.

[5] F. Voelker and G. Lambertson, Report No. BECON-94, ABC-27, 1991. 
[6] D. Teytelman, http://www.slac.stanford.edu/ dim/lecture1.pdf, 2003.

[7] D. Teytelman et al., in Proceedings of the IEEE Particle Accelerator Conference, Knoxville, TN, 2005; SLACPUB-11252.

[8] D. Teytelman et al., in Proceedings of the IEEE Particle Accelerator Conference, Vancouver, British Columbia, Canada, 1997; SLACPUB-8415.

[9] D. Teytelman et al., in Proceedings of the European Particle Accelerator Conference, Lucerne, 2004 (EPSAG, Lucerne, 2004).

[10] U. Wienands et al., in Proceedings of the IEEE Particle Accelerator Conference, Albuquerque, NM, 2007; SLAC-
PUB-12612

[11] A. Novokhatski, in Proceedings of the IEEE Particle Accelerator Conference, Albuquerque, NM, 2007; SLAC-PUB-12653.

[12] SLAC-PUB-418, 1993.

[13] J. Fox et al., in Proceedings of the IEEE Particle Accelerator Conference, Albuquerque, NM, 2007; SLAC-PUB-12636.

[14] D. Teytelman, in Proceedings of the IEEE Particle Accelerator Conference, Knoxville, TN, 2005; SLACPUB-11252, 2005.

[15] F. Pedersen, IEEE Trans. Nucl. Sci. 32, 2138 (1985). 